\title{
Genomic profiling of malignant peritoneal mesothelioma reveals recurrent alterations in epigenetic regulatory genes BAP1, SETD2, and DDX3X
}

\author{
Nancy M Joseph ${ }^{1,2}$, Yunn-Yi Chen ${ }^{1}$, Anthony Nasr ${ }^{3}$, Iwei Yeh ${ }^{1,2}$, Eric Talevich ${ }^{2}$, \\ Courtney Onodera ${ }^{2}$, Boris C Bastian ${ }^{1,2}$, Joseph T Rabban ${ }^{1}$, Karuna Garg ${ }^{1}$, Charles Zaloudek ${ }^{1}$ \\ and David A Solomon ${ }^{1,2}$ \\ ${ }^{1}$ Department of Pathology, University of California, San Francisco, CA, USA; ${ }^{2}$ Clinical Cancer Genomics \\ Laboratory, University of California, San Francisco, CA, USA and ${ }^{3}$ Pathology Sciences Medical Group, Chico, \\ CA, USA
}

\begin{abstract}
Malignant mesothelioma is a rare cancer that arises from the mesothelial cells that line the pleural cavity and less commonly from the peritoneal lining of the abdomen and pelvis. Most pleural mesotheliomas arise in patients with a history of asbestos exposure, whereas the association of peritoneal mesotheliomas with exposure to asbestos and other potential carcinogens is less clear, suggesting that the genetic alterations that drive malignant peritoneal mesothelioma may be unique from those in pleural mesothelioma. Treatment options for all malignant mesotheliomas are currently limited, with no known targeted therapies available. To better understand the molecular pathogenesis of malignant peritoneal mesothelioma, we sequenced 510 cancer-related genes in 13 patients with malignant mesothelioma arising in the peritoneal cavity. The most frequent genetic alteration was biallelic inactivation of the BAP1 gene, which occurred in $9 / 13$ cases, with an additional two cases demonstrating monoallelic loss of BAP1. All 11 of these cases demonstrated loss of BAP1 nuclear staining by immunohistochemistry, whereas two tumors without BAP1 alteration and all 42 cases of histologic mimics in peritoneum ( 8 multilocular peritoneal inclusion cyst, 6 well-differentiated papillary mesothelioma of the peritoneum, 16 adenomatoid tumor, and 12 low-grade serous carcinoma of the ovary) demonstrated intact BAP1 nuclear staining. Additional recurrently mutated genes in this cohort of malignant peritoneal mesotheliomas included NF2 (3/13), SETD2 (2/13), and DDX3X (2/13). While these genes are known to be recurrently mutated in pleural mesotheliomas, the frequencies are distinct in peritoneal mesotheliomas, with nearly $85 \%$ of peritoneal tumors harboring $B A P 1$ alterations versus only $20-30 \%$ of pleural tumors. Together, these findings demonstrate the importance of epigenetic modifiers including BAP1, SETD2, and DDX3X in mesothelial tumorigenesis and suggest opportunities for targeted therapies.

Modern Pathology (2017) 30, 246-254; doi:10.1038/modpathol.2016.188; published online 4 November 2016
\end{abstract}

Malignant mesothelioma is a rare cancer that arises from the mesothelial cells that line the pleural cavity ( $>80 \%$ of cases) and less commonly from the peritoneal lining of the abdominal and pelvic

Correspondence: Dr NM Joseph, MD, PhD, Department of Pathology, University of California, San Francisco, 1600 Divisadero Street, Room B-620, PO Box 0785, San Francisco, CA 94143, USA or Dr DA Solomon, MD, PhD, Department of Pathology, University of California, San Francisco, 513 Parnassus Ave, PO Box 0102, Health Sciences West 451, San Francisco, CA 94143, USA.

E-mail: nancy.joseph@ucsf.edu or david.solomon@ucsf.edu Received 2 August 2016; revised 18 September 2016; accepted 18 September 2016; published online 4 November 2016 cavities (10-15\%), the pericardial lining, and the tunica vaginalis. Approximately 2500 malignant mesotheliomas are newly diagnosed in the United States each year, which have a uniformly poor prognosis and median survival time of $<2$ years. Greater than $80 \%$ of malignant pleural mesotheliomas arise in patients with a history of asbestos exposure, whereas malignant peritoneal mesotheliomas have less clear risk factors that include chronic peritonitis. ${ }^{1}$ Patients with malignant peritoneal mesothelioma are more commonly female, of younger age, and have better prognosis than patients with pleural mesothelioma, though most patients will eventually die of disease. ${ }^{2}$ 
Genomic analysis has defined the spectrum of molecular alterations that drive pleural mesothelioma. These include deletion of the CDKN2A tumor suppressor gene ( $>60 \%$ of cases) that encodes the cell cycle kinase inhibitor protein $\mathrm{p} 16^{\mathrm{INK} 4 \mathrm{a}}$, mutation of the NF2 tumor suppressor gene $(20-40 \%$ of cases) that encodes the cytoskeletal scaffolding protein Merlin, and mutation or deletion of the BAP1 tumor suppressor gene (20-30\% of cases) that encodes a deubiquitinating enzyme originally identified as a BRCA1 interacting protein. ${ }^{3-7}$ Most recently, four distinct molecular subtypes were identified that correlated with epithelioid, sarcomatoid, biphasicepithelioid, and biphasic-sarcomatoid histologies, with the epithelioid subtype showing the longest survival times. ${ }^{7}$ Inactivating mutations in two epigenetic regulatory genes, $D D X 3 X$ and SETD2, were identified in a 4 and $8 \%$ of pleural mesotheliomas, respectively. ${ }^{7}$ In addition to these somatic mutations found in sporadic mesotheliomas, germline mutation of $B A P 1$ has been identified in rare patients with malignant mesothelioma as part of the $B A P 1$ tumor predisposition syndrome (OMIM 603089), which in addition to mesothelioma includes increased incidence of uveal melanoma, meningioma, renal cell carcinoma, and other tumors. ${ }^{8,9}$ Given the frequent genetic inactivation of $C D K N 2 A$ and $B A P 1$ in malignant pleural mesothelioma, several recent studies have assessed the sensitivity and specificity of either fluorescence in situ hybridization or imunohistochemistry against these two genes/proteins as helpful diagnostic markers to distinguish malignant mesothelioma from benign mesothelial proliferations and metastatic carcinoma in pleural fluid. ${ }^{10-14}$ BAP1 immunostaining has clearly emerged as a useful adjunct for the cytopathologist in characterizing mesothelial proliferations in the pleural cavity.

The genetic alterations which drive malignant mesothelioma in the peritoneum and other body cavities have been speculated to potentially be distinct from pleural mesotheliomas but had not been investigated until very recently. Indeed, one study performed fluorescence in situ hybridization on mesotheliomas from different sites of origin and found chromosome 9p21 deletion much more commonly in pleural than peritoneal tumors (85 vs 36\%), whereas 5 p15 and 7 p12 gains were more common in peritoneal than pleural tumors. ${ }^{15}$ A recent genomewide analysis of 12 peritoneal mesotheliomas found $B A P 1$ alterations (either deletions or truncating mutations) in 7 cases, but failed to identify alterations in CDKN2A or NF2 that are common in pleural mesotheliomas. ${ }^{16}$ Another recent study correlating genetic status with outcomes for patients with peritoneal mesothelioma identified frequent loss of BAP1 immunostaining that did not correlate with significant differences in outcome, but did find that tumors harboring both CDKN2A homozygous deletion and NF2 hemizygous loss were associated with worse progression-free and overall survival. ${ }^{17}$ The specificity of BAP1 loss in mesothelial proliferations and histologic mimics of malignant mesothelioma in the peritoneum including serous carcinoma of the ovary is currently under investigation and has shown promising results in the limited investigations performed to date. ${ }^{12,13,18}$

Given their uniformly poor prognosis, lack of well-established risk factors, and poorly understood pathogenesis, we sought to better define the genetic events that define malignant peritoneal mesothelioma and potentially identify targetable alterations to ultimately improve outcomes through a combination of next-generation sequencing and immunohistochemical assays.

\section{Materials and methods}

\section{Study Population}

This study was approved by the Institutional Review Board of the University of California, San Francisco. Thirteen cases of primary malignant peritoneal mesotheliomas were retrieved from the archives of the University of California, San Francisco Department of Pathology, spanning years 1991-2015. All 13 cases were confined to the abdomen and pelvis and had no evidence of pleural involvement. All cases were re-reviewed by three gynecologic pathologists who agreed with the diagnosis of malignant mesothelioma. All 13 cases demonstrated epithelioid morphology, while one case also had a focal sarcomatoid component. Cases of multilocular peritoneal inclusion cyst also known as benign multicystic mesothelioma $(n=8)$, well-differentiated papillary mesothelioma of the peritoneum $(n=6)$, adenomatoid tumor $(n=16)$, and low-grade serous carcinoma of the ovary $(n=12)$ were also retrieved from the archives of the University of California, San Francisco Department of Pathology, spanning years 2000-2015. All specimens were fixed in 10\% neutral buffered formalin and embedded in paraffin. Clinical information on patients with malignant peritoneal mesothelioma was obtained from electronic medical records. Selected histologic and clinicopathologic parameters were recorded, including patient age at diagnosis, location of tumor, type of surgical procedure, treatment, and outcome.

\section{Capture-Based Next-Generation DNA Sequencing}

Genomic DNA was extracted from formalin-fixed paraffin-embedded tissue blocks of matched normal and tumor tissues of 13 patients with malignant peritoneal mesothelioma using the QIAamp DNA FFPE Tissue Kit (Qiagen). Capture-based next-generation sequencing was performed at the University of California, San Francisco Clinical Cancer Genomics Laboratory, using an assay that targets the coding regions of 510 cancer-related genes, select introns of 40 genes, and TERT promoter with a total 
sequencing footprint of $2.8 \mathrm{Mb}$ (UCSF500 Cancer Gene Panel). Sequencing libraries were prepared from genomic DNA. Target enrichment was performed by hybrid capture using a custom oligonucleotide library. Sequencing was performed on an Illumina HiSeq 2500. Duplicate sequencing reads were removed computationally to allow for accurate allele frequency determination and copy-number calling. The analysis was based on the human reference sequence (NCBI build 37) using the following software packages: BWA, Samtools, Picard tools, GATK, CNVkit, Pindel, SATK, Annovar, Freebayes, Delly, and Nexus Copy Number. ${ }^{19-26}$

\section{Immunohistochemistry}

Immunohistochemistry for BAP1 was performed on whole-tissue sections of cases of malignant peritoneal mesothelioma $(n=13)$, well-differentiated papillary mesothelioma of the peritoneum $(n=6)$, multilocular peritoneal inclusion cyst $(n=8)$, adenomatoid tumor $(n=16)$, and low-grade serous carcinoma of the ovary $(n=12)$. A monoclonal BAP1 antibody (clone C-4, Santa Cruz Biotechnology, sc28383) was used at 1:100 dilution following ER2 antigen retrieval on a Leica Bond autostainer. BAP1 staining was scored as either intact (strong homogeneous nuclear staining of $>90 \%$ of tumor cells) or lost (no nuclear staining in tumor cells with intact expression in non-neoplastic stromal and endothelial cells).

\section{Results}

\section{Histologic and Clinical Features of Malignant Peritoneal Mesotheliomas}

Thirteen cases of malignant mesothelioma confined to the abdomen and pelvis without pleural involvement were retrieved from our institutional pathology archives. These included eight female patients (ages 19-89) and five male patients (ages 53-71). Twelve of the 13 cases demonstrated a predominantly epithelioid morphology, while one case had mixed epithelioid and sarcomatoid morphology. Limited clinical outcome data was available for the 13 cases, which is summarized in Table 1.

\section{Genomic Alterations in Malignant Peritoneal Mesotheliomas}

Targeted next-generation sequencing of 510 cancerassociated genes revealed pathogenic genetic alterations in each of the 13 cases (Figure 1; Supplementary Table 1). BAP1 alterations were identified in 11 of 13 tumors (85\%). Of these 11 cases, three had somatic BAP1 mutations accompanied by loss of heterozygosity (one with a frameshift mutation, p.D362fs; one with a splice site mutation,
c.68-1G $>$ A; and one with a missense mutation, p.L97Q). One case had two somatic BAP1 mutations (p.H669D and p.86_87del). Additionally, two cases had focal homozygous deletions of the BAP1 locus, and two cases had structural rearrangements involving BAP1 accompanied by loss of heterozygosity. Two cases demonstrated heterozygous loss of the BAP1 locus on chromosome $3 p$ but lacked an identifiable alteration of the second allele. The final case had a germline frameshift mutation in BAP1 (p.L573fs) that was accompanied by loss of heterozygosity in the tumor. Of the 9 cases with biallelic $B A P 1$ alterations, two cases also demonstrated inactivating frameshift mutations (p.V2345fs and p.Q1152fs) in the epigenetic regulatory gene SETD2 with accompanying loss of heterozygosity. One of these tumors also harbored a truncating mutation (p. L1051fs) in the ARID1A gene, which encodes a subunit of the Swi/Snf chromatin remodeling complex. In addition, two other cases with biallelic $B A P 1$ alteration demonstrated mutations in another epigenetic regulatory gene, $D D X 3 X$. Three tumors harbored inactivating mutations in the NF2 tumor suppressor gene (p.Q428*, p.F47fs, and p.L176fs) including both of the two cases with heterozygous $B A P 1$ loss and one case without BAP1 alteration or chromosome $3 \mathrm{p}$ loss. These three truncating mutations in NF2 were each accompanied by loss of heterozygosity. In the remaining case without BAP1 or NF2 alteration, a hotspot mutation in the TERT promoter (chr5: g.1,295,228G >A) was the solitary pathogenic alteration identified. The two tumors without BAP1 alteration occurred in the oldest (age 85) and youngest (age 19) patients in this cohort and each demonstrated a solitary genetic alteration, NF2 truncating mutation with loss of heterozygosity in the 85-year old and TERT promoter mutation in the 19-year old. The one case of peritoneal mesothelioma with mixed epithelioid and sarcomatoid morphology was the only case found to harbor homozygous deletion of CDKN2A and focal high-level amplification of the MET oncogene in addition to a structural rearrangement involving $B A P 1$.

\section{BAP1 Immunohistochemistry Can Aid in the Diagnosis of Malignant Peritoneal Mesothelioma}

BAP1 immunohistochemistry was performed on all 13 malignant peritoneal mesotheliomas (Figure 2; Table 2), as well as a series of tumors commonly considered in the differential diagnosis of malignant peritoneal mesothelioma including 6 cases of well-differentiated papillary mesothelioma, 8 cases of multicystic peritoneal inclusion cyst, 16 cases of adenomatoid tumor, and 12 cases of low-grade serous carcinoma of the ovary (Figure 3; Table 2). Loss of nuclear staining for BAP1 protein was seen in 11 of the 13 peritoneal mesotheliomas (85\%), all of which demonstrated genetic alteration of the 
Table 1 Clinical data for patients with malignant peritoneal mesothelioma

\begin{tabular}{|c|c|c|c|c|c|}
\hline Patient & Age at $d x$ & Sex & Year of $d x$ & Presenting symptoms & Clinical outcome data \\
\hline MPM1 & 74 & $\mathrm{~F}$ & 2009 & Abdominal distention & $\begin{array}{l}\text { Diagnosed on paracentesis followed by hysterctomy/bilateral salpingo-oophorectomy/omentectomy/ } \\
\text { debulking, no follow-up available after surgery }\end{array}$ \\
\hline MPM2 & 89 & $\mathrm{~F}$ & 2008 & Bowel obstruction & Diagnosed on bilateral oophorectomy/myomectomy/mesenteric biopsy, no follow-up available after surgery \\
\hline MPM3 & 48 & $\mathrm{~F}$ & 1996 & Pelvic pain & $\begin{array}{l}\text { Initially underwent hysterectomy/bilateral salpingo-oophorectomy with atypical mesothelial proliferation, } \\
7 \text { months later underwent diagnostic surgical resection of } 10 \mathrm{~cm} \text { pelvic mass and left colectomy, received } \\
\text { intraperitoneal chemotherapy with cisplatin/doxorubicin/taxol, underwent radiation therapy, underwent two } \\
\text { additional debulking surgeries at one and two after diagnosis, presented with bowel obstruction at three years } \\
\text { after diagnosis due to recurrent disease, referred to hospice care without additional treatment }\end{array}$ \\
\hline MPM4 & 47 & $\mathrm{~F}$ & 2008 & Vaginal bleeding & $\begin{array}{l}\text { Diagnosed on hysterectomy/bilateral salpingo-oophorectomy/omentectomy/debulking, metastatic disease to } \\
\text { cervical lymph nodes confirmed by biopsy at time of diagnosis, received } 8 \text { cycles of adjuvant chemotherapy } \\
\text { with pemetrexed and cisplatin, imaging showing recurrent disease at one year after surgery, began additional } \\
\text { treatment with gemcitabine and vinorelbine, no additional follow-up available }\end{array}$ \\
\hline MPM5 & 48 & M & 1993 & Umbilical hernia & $\begin{array}{l}\text { Diagnosed on herniorrhaphy followed by debulking surgery, received adjuvant intraperitoneal chemotherapy, } \\
\text { underwent repeat debulking surgeries at two and five years after diagnosis, no additional follow-up available } \\
\text { after last debulking surgery }\end{array}$ \\
\hline MPM6 & 54 & M & 2004 & Abdominal distention & $\begin{array}{l}\text { Diagnosed on paracentesis, received } 6 \text { cycles of neoadjuvant chemotherapy with pemetrexed and cisplatin, } \\
\text { then underwent debulking surgery with right colectomy, no follow-up available after surgery }\end{array}$ \\
\hline MPM7 & 67 & $\mathrm{M}$ & 1991 & Abdominal wall mass & Diagnosed on resection of mass and involved small bowel, no follow-up available after surgery \\
\hline MPM8 & 55 & M & 2000 & $\begin{array}{l}\text { Abdominal distention and } \\
\text { ascites }\end{array}$ & $\begin{array}{l}\text { Initially had five non-diagnostic paracentesis cytologic evaluations, diagnosed on laparoscopy with omental } \\
\text { biopsy followed by omentectomy and surgical debulking, no follow-up available after surgery }\end{array}$ \\
\hline MPM9 & 62 & $\mathrm{~F}$ & 2014 & Pelvic mass & $\begin{array}{l}\text { Diagnosed on hysterectomy/bilateral salpingo-oophorectomy/omentectomy/colectomy, no follow-up available } \\
\text { after surgery }\end{array}$ \\
\hline MPM10 & 71 & $\mathrm{M}$ & 2002 & Abdominal distention & Diagnosed on omentectomy and debulking surgery, no follow-up available after surgery \\
\hline MPM11 & 70 & $\mathrm{~F}$ & 2014 & $\begin{array}{l}\text { Abdominal distention and } \\
\text { ascites }\end{array}$ & Diagnosed on laparoscopy with omental biopsy, no follow-up available after biopsy \\
\hline MPM12 & 85 & $\mathrm{~F}$ & 2006 & $\begin{array}{l}\text { Abdominal distention and } \\
\text { ascites }\end{array}$ & $\begin{array}{l}\text { Diagnosed on hysterectomy/bilateral salpingo-oophorectomy/omental biopsy, no follow-up available after } \\
\text { surgery }\end{array}$ \\
\hline MPM13 & 19 & $\mathrm{~F}$ & 2004 & Abdominal distention & $\begin{array}{l}\text { Diagnosed on bilateral salpingo-oophorectomy/omentectomy/surgical debulking, received adjuvant } \\
\text { chemotherapy with } 4 \text { cycles of pemetrexed and cisplatin, developed recurrent disease four years after } \\
\text { diagnosis, received } 3 \text { cycles of pemetrexed and carboplatin, underwent hysterectomy and surgical debulking, } \\
\text { treated with gemcitabine then vinorelbine then liposomal doxorubicin, died of disease } 6 \text { years after diagnosis }\end{array}$ \\
\hline
\end{tabular}



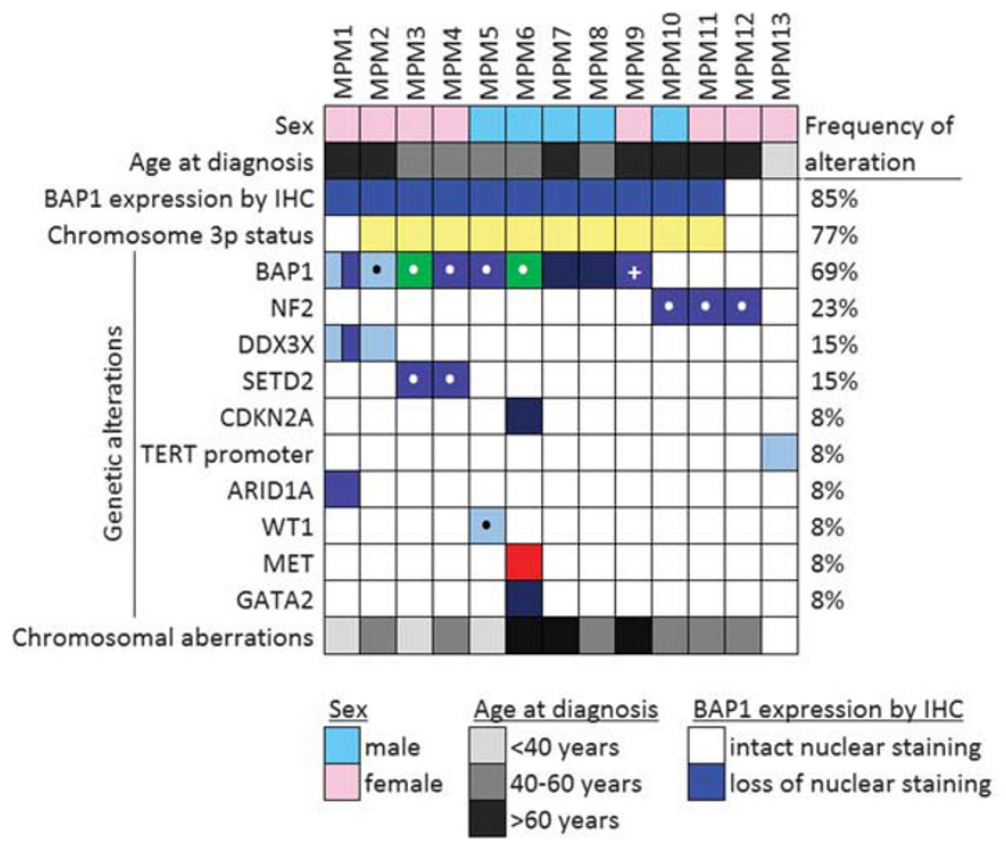

Chromosome 3p status
$\begin{aligned} & \square \text { Diploid } \\ & \text { Haploid or interstitial loss }\end{aligned}$
Chromosomal gains/losses
$\begin{aligned} & \text { Zero } \\ & 1-3 \\ & 4-9 \\ & 10+\end{aligned}$

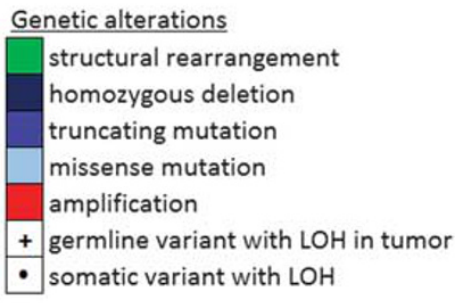

Figure 1 Genomic profiling results on 13 patients with malignant peritoneal mesothelioma. Patient age, sex, tumor histologic subtype, chromosome $3 p$ status, BAP1 nuclear immunostaining results, pathogenic or likely pathogenic genetic alterations, and the quantity of chromosomal copy-number aberrations are shown.

$B A P 1$ gene. In all 11 of these tumors, retained BAP1 expression was present in non-neoplastic stromal, inflammatory, and endothelial cells. Moreover, BAP1 loss was seen uniformly throughout all tumor cells in each case, suggesting that BAP1 inactivation is an early or initiating event in mesothelial tumorigenesis. The absence of nuclear BAP1 protein in the two tumors with only heterozygous loss of the $B A P 1$ locus suggests a high likelihood of an unidentified alteration involving the second allele and potentially indicates that BAP1 immunohistochemistry may be a more sensitive diagnostic test for $B A P 1$ inactivation compared with capture-based next-generation sequencing assay. Intact nuclear staining for BAP1 protein was present in 2 of the 13 peritoneal mesotheliomas, both of which demonstrated two wild-type copies of BAP1 by our sequencing assay. All 42 cases of welldifferentiated papillary mesothelioma of the peritoneum, multilocular peritoneal inclusion cyst, adenomatoid tumor, and low-grade serous carcinoma of the ovary demonstrated intact BAP1 nuclear staining.

\section{Discussion}

Our study reveals that while a similar spectrum of genetic alterations define both pleural and peritoneal mesothelioma, these alterations occur at distinct frequencies depending on the anatomic origin of the tumor. Although CDKN2A deletion is very common in pleural mesotheliomas, it was only seen in one of 13 peritoneal mesotheliomas, which was the only case with a sarcomatoid component. In contrast, BAP1 inactivation/loss is much more common in peritoneal than pleural mesotheliomas. DDX $3 X$ and SETD2 mutations appear to be present in a small subset (5-15\%) of both pleural and peritoneal mesotheliomas. A recent array comparative genomic hybridization study on 33 malignant peritoneal mesotheliomas identified focal deletions affecting $B A P 1$ ( $61 \%$ of cases), NF2 (21\% of cases), and $C D K N 2 A$ (33\% of cases), providing additional support for our conclusion that similar genetic alterations drive peritoneal mesothelioma but at different frequency compared with pleural mesothelioma. ${ }^{27}$ 

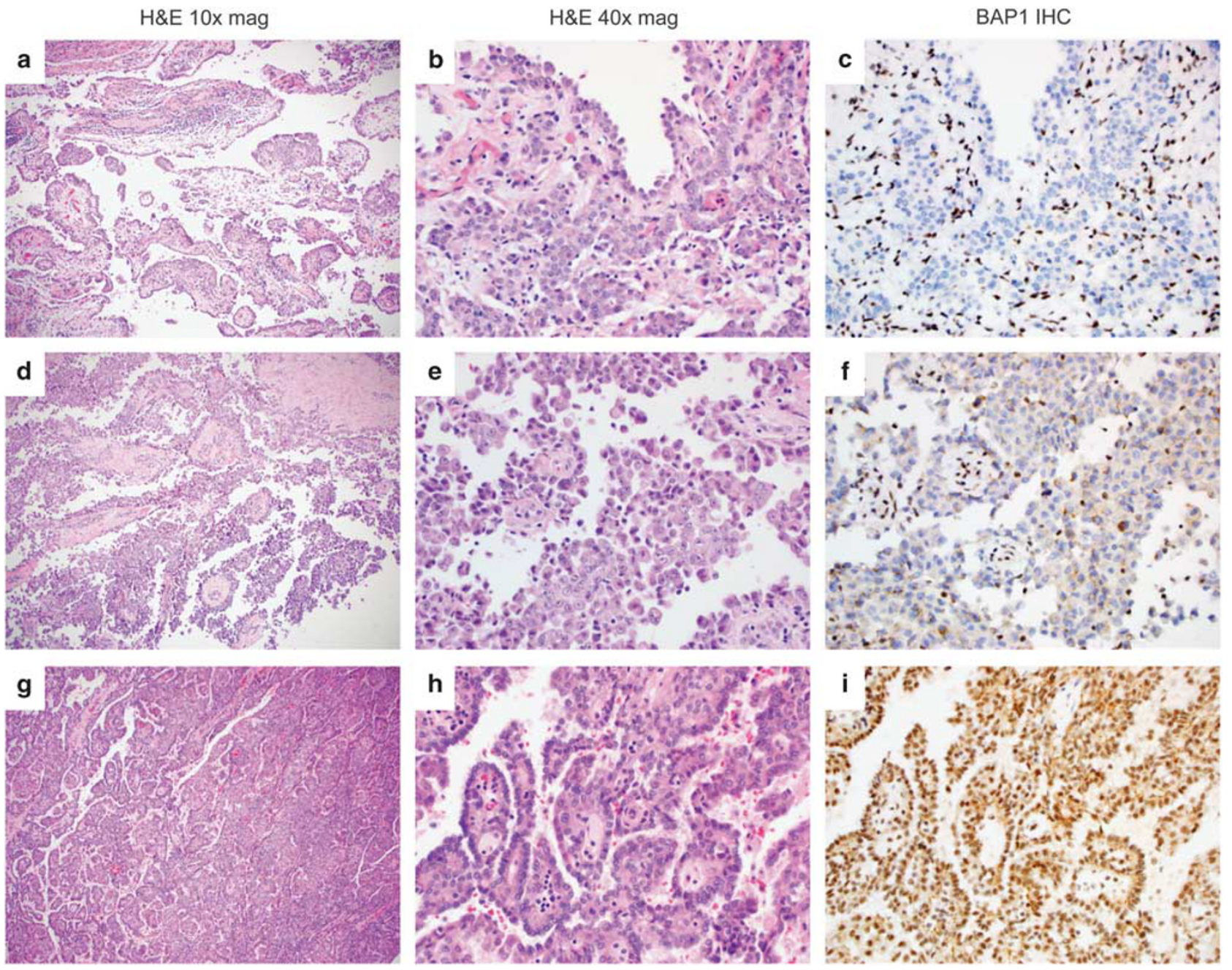

Figure 2 Loss of nuclear BAP1 expression defines the majority of malignant peritoneal mesotheliomas arising within the abdomen and pelvis. (a-c) Absence of BAP1 immunostaining in a malignant mesothelioma arising in the abdomen/pelvis of a 70-year old woman (patient MPM11) with interstitial deletion of chromosome 3p encompassing the BAP1 locus, as well as an NF2 frameshift mutation. Retained BAP1 expression is seen in non-neoplastic stromal, inflammatory, and endothelial cells. (d-f) Absence of BAP1 immunostaining in a malignant mesothelioma arising in the abdomen/pelvis of a 67-year old man (patient MPM7) with focal homozygous deletion of a $300 \mathrm{~kb}$ segment on chromosome $3 \mathrm{p}$ encompassing the BAP1 locus. (g-i) Intact BAP1 immunostaining in a malignant mesothelioma arising in the abdomen/pelvis of a 19-year old woman (patient MPM13) with hotspot mutation in the TERT promoter, without chromosome $3 \mathrm{p}$ loss or BAP1 alteration.

Table 2 BAP1 immunostaining results on malignant peritoneal mesotheliomas and histologic mimics in the peritoneum

\begin{tabular}{|c|c|c|c|}
\hline Tumor type & $\begin{array}{l}\text { Cases with loss of nuclear BAP1 } \\
\text { immunostaining }\end{array}$ & $\begin{array}{l}\text { Total number } \\
\text { of cases }\end{array}$ & $\begin{array}{c}\% \text { With BAP1 } \\
\text { loss }\end{array}$ \\
\hline Malignant peritoneal mesothelioma & 11 & 13 & 85 \\
\hline Ovarian low-grade serous carcinoma & 0 & 12 & 0 \\
\hline Well-differentiated papillary mesothelioma & 0 & 6 & 0 \\
\hline Multilocular peritoneal inclusion cyst & 0 & 8 & 0 \\
\hline Adenomatoid tumor & 0 & 16 & 0 \\
\hline
\end{tabular}

Our study also provides further support for the specificity of BAP1 protein loss as a helpful diagnostic marker for the pathologic identification of malignant peritoneal mesotheliomas. ${ }^{10-14}$ We observed intact BAP1 expression in all 42 cases of potential histologic mimics in the abdomen and pelvis including well-differentiated papillary mesothelioma, multilocular peritoneal inclusion cyst, adenomatoid tumor, and peritoneal metastases of ovarian low-grade serous carcinoma.

Finally, our genomic profiling of malignant peritoneal mesothelioma has identified recurrent 

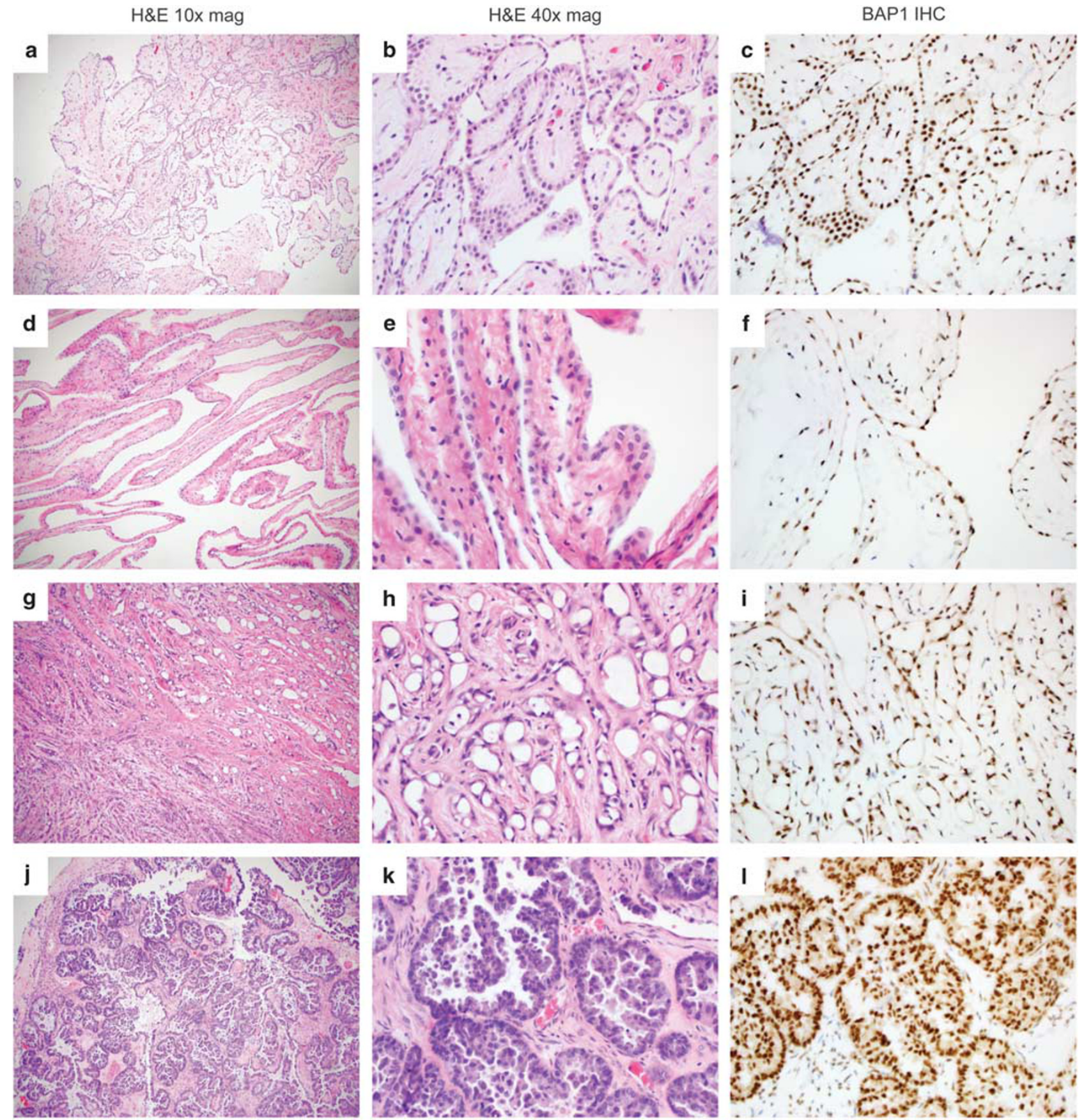

Figure 3 Intact BAP1 expression is uniformly present in benign mesothelial lesions and other histologic mimics of malignant mesothelioma in the peritoneum. (a-c) Intact BAP1 immunostaining in a well-differentiated papillary mesothelioma within the abdomen of a 67-year old woman who presented with gastric volvulus and was found to have peritoneal nodularity along the stomach and liver. (d-f) Intact BAP1 immunostaining in an $8 \mathrm{~cm}$ multilocular peritoneal inclusion cyst in the pelvic cavity of a 62-year old woman. (g-i) Intact BAP1 immunostaining in a $2 \mathrm{~cm}$ paratesticular adenomatoid tumor in a 43-year old man who underwent orchiectomy. (j-l) Intact BAP1 immunostaining in an ovarian low-grade serous carcinoma in an 83-year old woman with multiple metastatic deposits throughout the abdomen and pelvis.

mutations in the epigenetic regulatory genes $B A P 1$, SETD2, and DDX3X. These findings suggest that transcriptional deregulation is a key oncogenic mechanism in mesothelial tumorigenesis and has important therapeutic implications for the treatment of this disease. Emerging preclinical evidence suggests that inhibitors of epigenetic modifiers including histone deacetylases and the histone methyltransferase EZH2 may be efficacious in the treatment of mesothelioma. ${ }^{28,29}$ Those mesotheliomas harboring inactivating events affecting both $B A P 1$ and other transcriptional regulators such as 
SETD2, DDX3X, and ARID1A likely define a molecular subgroup with altered transcriptional programs that may respond favorably to these agents, compared with those mesotheliomas lacking these alterations. Additional potentially targetable alterations that emerged from our study include small molecule inhibition of MET (eg, cabozantinib) for the subset of peritoneal mesotheliomas harboring MET amplification, as well as small molecule inhibition of WEE1 (eg, AZD1775) for the subset of tumors harboring SETD2 inactivation, a synthetic lethality that was recently reported in renal cell carcinoma and osteosarcoma. ${ }^{30}$

\section{Acknowledgments}

This study was funded by the UCSF Department of Pathology. DAS is additionally supported by an NIH Director's Early Independence Award (DP5 OD021403).

\section{Disclosure/conflict of interest}

The authors declare no conflict of interest.

\section{References}

1 Robinson BWS, Lake RA. Advances in malignant mesothelioma. N Engl J Med 2005;353: 1591-1603.

2 Liu S, Staats P, Lee M, et al. Diffuse mesothelioma of the peritoneum: correlation between histological and clinical parameters and survival in 73 patients. Pathology 2014;46:604-609.

3 Bianchi AB, Mitsunaga SI, Cheng JQ, et al. High frequency of inactivating mutations in the neurofibromatosis type gene (NF2) in primary malignant mesotheliomas. Proc Natl Acad Sci USA 1995;92: 10854-10858.

4 Sekido Y, Pass HI, Bader S, et al. Neurofibromatosis type 2 (NF2) gene is somatically mutated in mesothelioma but not in lung cancer. Cancer Res 1995;55: 1227-1231.

5 Bott M, Brevet M, Taylor BS, et al. The nuclear deubiquitinase BAP1 is commonly inactivated by somatic mutations and 3p21.1 losses in malignant pleural mesothelioma. Nat Genet 2011;43:668-672.

6 Guo G, Chmielecki J, Goparaju C, et al. Whole-exome sequencing reveals frequent genetic alterations in BAP1, NF2, CDKN2A, and CUL1 in malignant pleural mesothelioma. Cancer Res 2015;75:264-269.

7 Bueno R, Stawiski EW, Goldstein LD, et al. Comprehensive genomic analysis of malignant pleural mesothelioma identifies recurrent mutations, gene fusions and splicing alterations. Nat Genet 2016;48: 407-416.

8 Testa JR, Cheung M, Pei J, et al. Germline BAP1 mutations predispose to malignant mesothelioma. Nat Genet 2011;43:1022-1025.

9 Abdel-Rahman MH, Pilarski R, Cebulla CM, et al. Germline BAP1 mutation predisposes to uveal melanoma, lung adenocarcinoma, meningioma, and other cancers. J Med Genet 2011;48:856-859.

10 Sheffield BS, Hwang HC, Lee AF, et al. BAP1 immunohistochemistry and p16 FISH to separate benign from malignant mesothelial proliferations. Am J Surg Pathol 2015;39:977-982.

11 McGregor SM, Dunning R, Hyjek E, et al. BAP1 facilitates diagnostic objectivity, classification, and prognostication in malignant pleural mesothelioma. Hum Pathol 2015;46:1670-1678.

12 Cigognetti M, Lonardi S, Fisogni S, et al. BAP1 (BRCA1-associated protein 1) is a highly specific marker for differentiating mesothelioma from reactive mesothelial proliferations. Mod Pathol 2015;28: 1043-1057.

13 Andrici J, Sheen A, Sioson L, et al. Loss of expression of BAP1 is a useful adjunct, which strongly supports the diagnosis of mesothelioma in effusion cytology. Mod Pathol 2015;28:1360-1368.

14 Hwang HC, Sheffield BS, Rodriguez S, et al. Utility of BAP1 immunohistochemistry and p16 (CDKN2A) FISH in the diagnosis of malignant mesothelioma in effusion cytology specimens. Am J Surg Pathol 2016;40: 120-126.

15 Takeda M, Kasai T, Enomoto Y, et al. Comparison of genomic abnormality in malignant mesothelioma by the site of origin. J Clin Pathol 2014;67:1038-1043.

16 Alakus H, Yost SE, Woo B, et al. BAP1 mutation is a frequent somatic event in peritoneal malignant mesothelioma. J Transl Med 2015;13:122.

17 Singhi AD, Krasinskas AM, Choudry HA, et al. The prognostic significance of BAP1, NF2, and CDKN2A in malignant peritoneal mesothelioma. Mod Pathol 2016;29:14-24.

18 Andrici J, Jung J, Sheen A, et al. Loss of BAP1 expression is very rare in peritoneal and gynecologic serous adenocarcinomas and can be useful in the differential diagnosis with abdominal mesothelioma. Hum Pathol 2016;51:9-15.

19 Li H, Durbin R. Fast and accurate long-read alignment with Burrows-Wheeler transform. Bioinformatics 2010;26:589-595.

20 Li H, Handsaker B, Wysoker A, et al. The sequence alignment/map format and SAMtools. Bioinformatics 2009;25:2078-2079.

21 Broad Institute. Picard http://broadinstitute.github.io/ picard/.

22 Talevich E, Shain AH, Botton T, et al. CNVkit: genomewide copy number detection and visualization from targeted DNA sequencing. PLoS Comput Biol 2016;12: e1004873.

23 Ye K, Schulz MH, Long Q, et al. Pindel: a pattern growth approach to detect break points of large deletions and medium sized insertions from pairedend short reads. Bioinformatics 2009;25:2865-2871.

24 Wang K, Li M, Hakonarson H. ANNOVAR: functional annotation of genetic variants from high-throughput sequencing data. Nucleic Acids Res 2010;38: e164-e164.

25 Garrison E, Marth G. Haplotype-based variant detection from short-read sequencing. arXiv 1207.3907v2 [q-bio. GN].

26 Rausch T, Zichner T, Schlattl A, et al. DELLY: structural variant discovery by integrated paired-end and splitread analysis. Bioinformatics 2012;28:i333-i339.

27 Chirac P, Maillet D, Lepretre F, et al. Genomic copy alterations in 33 malignant peritoneal mesothelioma 
analyzed by comparative genomic hybridization array. Hum Pathol 2016;55:72-82.

28 Sacco JJ, Kenyani J, Butt Z, et al. Loss of the deubiquitylase BAP1 alters class I histone deacetylase expression and sensitivity of mesothelioma cells to HDAC inhibitors. Oncotarget 2015;6:13757-13771.
29 LaFave LM, Beguelin W, Koche R, et al. Loss of BAP1 function leads to EZH2-dependent transformation. Nat Med 2015;21:1344-1349.

30 Pfister SX, Markkanen E, Jiang Y, et al. Inhibiting WEE1 selectively kills histone H3K36me3-deficient cancers by dNTP starvation. Cancer Cell 2015;28:557-568.

Supplementary Information accompanies the paper on Modern Pathology website (http://www.nature.com/ modpathol) 